\title{
Profiling of the calcitonin-calcitonin receptor axis in primary prostate cancer: Clinical implications and molecular correlates
}

\author{
ARVIND THAKKAR ${ }^{1}$, IRENE V. BIJNSDORP ${ }^{2}$, ALBERT A. GELDOF ${ }^{2}$ and GIRISH V. SHAH ${ }^{1}$ \\ ${ }^{1}$ Department of Pharmacology, University of Louisiana, College of Pharmacy, Monroe, LA 71291, USA; \\ ${ }^{2}$ Department of Urology, VU University Medical Center, Amsterdam, The Netherlands
}

Received April 15, 2013; Accepted June 6, 2013

DOI: $10.3892 /$ or.2013.2583

\begin{abstract}
Expression of the neuroendocrine peptide calcitonin (CT) and its receptor (CTR) is frequently elevated in prostate cancers (PCs), and activation of the CT-CTR axis in noninvasive PC cells induces an invasive phenotype. We aimed to link CT/CTR expression in prostate specimens to clinicopathological parameters of PC. We analyzed CT and CTR expression in cohorts of benign prostates and primary PCs with/without metastatic disease by immunohistochemistry. Furthermore, we correlated CT/CTR expression with several clinicopathological parameters. CT/CTR immunostaining in benign prostate acini was predominantly localized to basal epithelium. However, this spatial specificity was lost in malignant prostates. PC sections displayed a remarkable increase in cell populations expressing CT/CTR and their staining intensity. Tumors with higher CT/CTR expression consistently displayed metastatic disease and poor clinical outcome. High CT/CTR expression in primary prostate tumors may serve as a prognostic indicator of disease aggressiveness and poor clinical outcome.
\end{abstract}

\section{Introduction}

Prostate cancer (PC) is the most commonly diagnosed cancer, and a second leading cause of cancer-related deaths in men in the Western world. Although PC typically undergoes slow growth, the tumor rapidly develops into aggressively growing and metastasizing cancer in a subset of men. Curative therapy for metastasized PC is not yet available (1). Although serum prostate specific antigen (PSA) test has improved the detection of PC considerably, it does not predict whether the tumor will

Correspondence to: Professor Girish V. Shah, Department of Pharmacology, College of Pharmacy, University of Louisiana, Monroe, LA 71291, USA

E-mail:shah@ulm.edu

Key words: calcitonin, calcitonin receptor, prostate cancer, prognostic indicator, metastasis remain indolent or will progress rapidly to a metastasizing cancer (2).

The calcitonin (CT) family of neuroendocrine peptides has gained attention as potential targets for therapeutic intervention in several cancer types (3-9). The members of this family include CT, adrenomedullin and CT gene-related peptide. CT receptor (CTR), a class B G protein-coupled receptor (GPCR) (10), maintains calcium homeostasis in the bone and the kidneys (11). It has also been implicated in early development, tissue repair and cancers $(12,13)$. The gene expression of CT and CTR is upregulated in advanced PC (9), suggesting a role for CT-CTR autocrine axis in tumor progression, invasion, angiogenesis and metastasis $(8,14,15)$. Consistent with this possibility, overexpression of CT and/or CTR in prostate cancer cells accelerates tumor growth and formation of distant metastases in athymic nude mice (8). In contrast, the knock-down of the expression of either CT or CTR reduces growth of aggressive prostate cancer cells and abolishes their ability to form distant metastases (14). However, no attempt has been made to link CT-CTR axis with clinicopathological parameters such as disease progression, metastasis or poor patient prognosis.

\section{Materials and methods}

Patient cohort description. Tissue samples from $61 \mathrm{PC}$ patients in the Department of Urology of the VU University Medical Center were obtained with the approval of the VU University Institutional Review Board. Median age of the patients was 64 years (range, 53-84) and median follow-up time was 67 months (range, 11-178). None of the patients received hormonal or radiation therapy prior to surgery. Tissue sections of each specimen were stained with hematoxylin and eosin and examined by a pathologist for histopathological diagnosis and tumor grading. Subject population was increased with the addition of 40 specimens of benign prostatic hyperplasia and 95 cores from 40 cases of advanced prostate cancer (TMA PR955; US Biomax Inc., Rockville, MD, USA). The clinicopathological data of each specimen including TNM stages, Gleason scores, preoperative serum PSA and survival data were also obtained. In all, 141 cases were examined. The cohorts were classified as follows: i) non-cancer, DRE negative, prostate biopsy negative; 
ii) cancer, organ-confined: Gleason Score: $3+3$, pT2a (absence of extracapsular extension or seminal vesicle invasion); iii) cancer, non-organ confined: Gleason Score $4+4$ or higher, pT3 (presence of extracapsular extension and/or seminal vesicle invasion); and iv) cancer, metastatic: confirmed distant metastases including hormone refractoriness.

Immunohistochemistry (IHC). Paraffin-embedded specimens were deparaffinized, hydrated, subjected to antigen retrieval by heating the slides for $5 \mathrm{~min}$ in $5 \mathrm{mM}$ sodium citrate. The sections were then stained for CT/CTR as previously described (14). Incubations with primary antibodies were followed by horseradish peroxidase phosphatase-conjugated secondary antibodies. The slides were then counterstained with hematoxylin.

Immunofluorescence. Antibodies against $\mathrm{CT}$ and the peptide (50-65 aa) of human CTR have been previously validated for the IHC of prostate cancer specimens (7). Tissue sections $(5 \mu \mathrm{m})$ were deparaffinized, hydrated and CT/CTR immunofluorescence was performed as previously described (14).

Controls. Tissue sections were incubated either in the presence of no primary antibody, no secondary antibody, or primary antibody blocked with the peptide.

Image analysis and interpretation. Six images per section were acquired. Immunostaining was scored by two individuals independently using established methods (16) and the mean reading was taken. The staining intensity was assigned an arbitrary value, on a scale of $0-3$ as follows: $(-), 0 ;(+/-), 0.5 ;(+)$, $1 ;(++) 2$; and (+++), 3 .

An IHC index for each sample was calculated by multiplying staining intensity with the percentage of positive cells. The results were graded from 0 (negative) to 300 (all cells display strong staining intensity). Reproducibility of the analysis was verified by rescoring of randomly chosen slides. Duplicate readings gave similar results.

The samples were broadly classified as: i) negative (IHC index $=0$ ); ii) low (IHC index <5); iii) moderate (IHC index $=5-50)$; iv) high (IHC index $=50-150)$; and v) very high (IHC index $>150$ ).

Statistical analysis. Statistical calculations were performed using Prism 5 computer program (GraphPad Software, San Diego, CA, USA). Results are generally expressed as mean IHC index \pm standard error of the mean (SEM) unless otherwise stated. $\mathrm{P}<0.05$ was considered to indicate a statistically significant result. One-way ANOVA and t-tests were used to compare CT/CTR IHC index across the clinical groups (cancer vs. non-cancer; organ-confined vs. metastatic; Gleason score 6 vs. $>8$ ). Receiver operating characteristics (ROC) curves were used to determine diagnostic utility of CT/CTR expression. Area under the curve (AUC) was calculated by numerical integration of each ROC curve. Cut-off points are defined as the values at which sensitivity and specificity are optimized. A two-sided P-value of $<0.05$ is considered to indicate a statistically significant result. Kaplan-Meier analysis was performed to compare the prognostic ability of CT/CTR expression with that of other clinicopathological parameters.
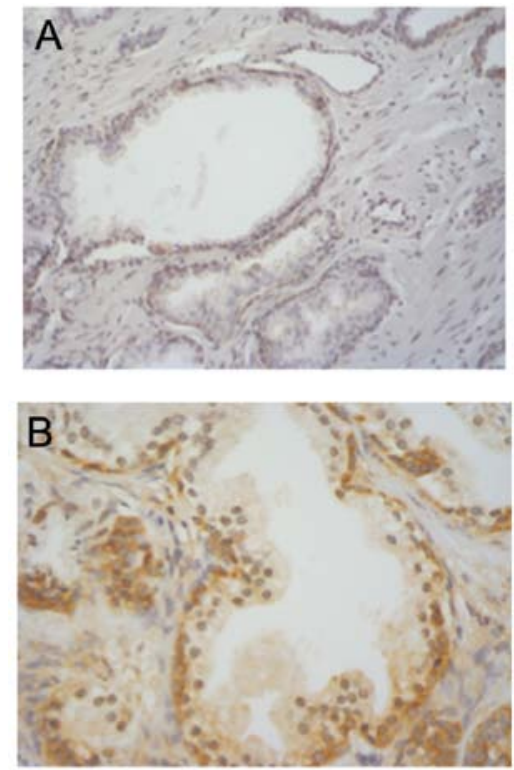

Figure 1. CT and CTR staining in benign and malignant prostates. (A) A typical x100 field of a benign prostate. In most cases, basal cells in benign acini stained focally and moderately for CT. However, in a minority of cases $(<15 \%)$, benign basal cells stained strongly and diffusely but secretory cells remained negative. (B) At a higher magnification (x400), it is evident that only basal cells stained for CT, but secretory cells remained negative.
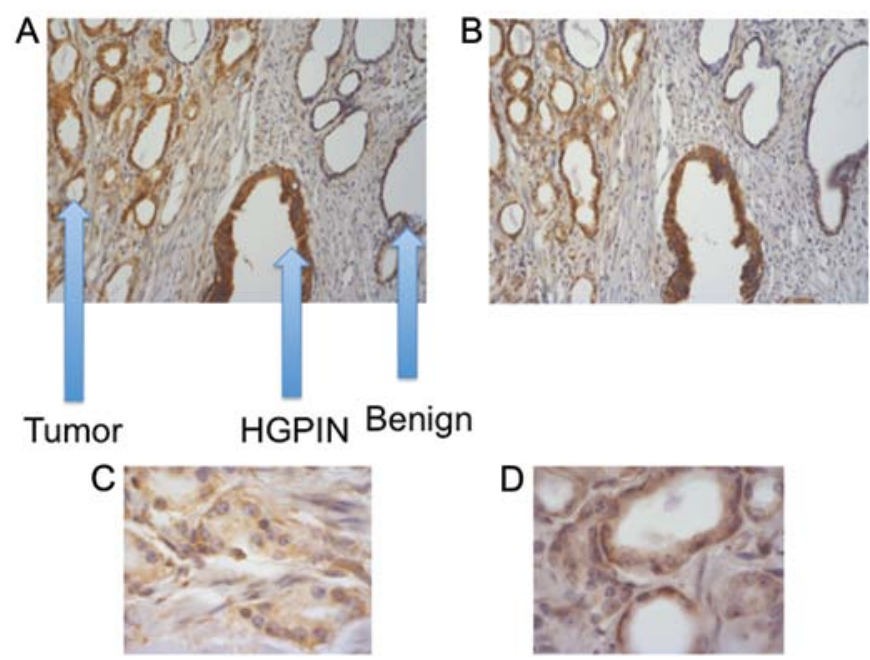

Figure 2. CT and CTR staining in benign, HGPIN and malignant regions of the prostate. PC sections of Gleason score $(3+3)$ were stained for (A) CT and (B) CTR. HGPIN and tumor showed strong CT and CTR staining. In contrast, staining for CT and CTR was very weak in benign regions (magnification, $\mathrm{x} 200$ ). (C) At x1000, cancer cells showed prominent nucleoli, also showed positive CT immunostaining. (D) Same as 3, but shows CTR staining.

\section{Results}

CT/CTR in the prostate. CT staining in benign acinii was predominantly localized to basal epithelium and was absent in secretory epithelium (Fig. 1). CTR expression displayed a similar profile (data not shown). Comparatively, CT/CTR immunostaining was absent/or weak in benign regions of the prostate, but was stronger in HGPIN and malignant acinii (Fig. 2A and B). Higher magnification images of a PC specimen suggest that basal epithelial cells displaying promi- 


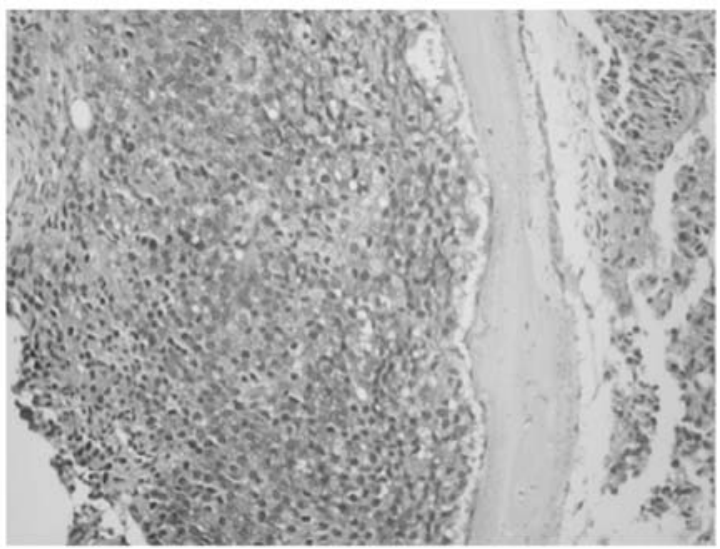

Figure 3. CT-immunopositive cells in bone metastasis of PC. A section of bone metastasis of a prostate cancer patient was stained for CT immunoreactivity (x100).

nent nucleoli were strongly CT- or CTR-positive (Fig. 2C and D). Bone metastasis of PC displayed intense cytoplasmic CT staining, suggesting tumor cells may secrete CT after implanting in the bone (Fig. 3).

Paracrine CT-CTR axis converts to autocrine in advanced PC. Since CT/CTR expression displayed a similar spatial localization, we evaluated whether CT and CTR are co-expressed in same cell populations. We performed double immunofluorescence-stainings of prostate TMA sections (TI95; US Biomax Inc.). A majority of cells in sections of PC of T stage 2 or 3 stained for either CT (red) or CTR (green) (Fig. 4A1-4). In contrast, a majority of CT-positive cells co-expressed CTR in $\mathrm{T}$ stage 4 cases as characterized by the yellow in the merged figure (Fig. 4B4 and C). These results, that CT-CTR axis is predominantly paracrine in earlier stages of $\mathrm{PC}$ but converts to autocrine in advanced stages, are consistent with our earlier results that activation of CT-CTR autocrine loop enhances the ability of PC cells to grow and metastasize $(8,14,17)$.
CT/CTR expression is upregulated in PC. Prostate specimens from pathology as well as TMA showed considerable variability in CT/CTR staining (Fig. 5). Specifically, benign specimens showed very weak stainings that increased to very strong in metastatic PCs.

The generated data of CT/CTR staining in benign prostates were pooled and compared with that from malignant prostates. Among 40 benign prostate specimens, 34 displayed negative staining for CT (85\%) and 6 displayed low staining (15\%). Among 101 primary PC specimens: 3 were negative $(<3 \%)$; 25 were low $(<25 \%)$; 28 were moderate $(<28 \%)$; 18 were high $(<18 \%)$; and 36 were very high $(<36 \%)$.

Significantly higher expression of CT and CTR in PC specimens was evident when assessed either as IHC index or as percent of immunopositive cells (Fig. 6A-D; $\mathrm{P}<0.0001$ for benign vs. adenocarcinoma). Interestingly, higher percentages of cells displayed CT expression than CTR expression. However, the intensity of CTR staining seemed stronger.

Is CT/CTR expression a prognostic marker for metastatic PC? We then stratified the data of PC specimens in two groups: localized PC and metastatic PC. The comparison of these two data sets show that metastatic PCs displayed significantly higher CT/CTR expression than localized PCs when assessed either as IHC index or as immunopositive cell populations (Fig. 6E-H). Stratification of the data further into T stages indicated that CT/CTR IHC indices of metastatic PCs in stages $2 \mathrm{c}$ and $3 \mathrm{a}$ were significantly higher than those of localized PCs in same $\mathrm{T}$ stages, raising a possibility that elevated CT/CTR expression in earlier $\mathrm{T}$ stages may be an indicator of metastatic disease (Fig. 6I and J).

ROC analysis of CT/CTR IHC index as a prognostic marker. We then analyzed the results by receiver operator characteristic (ROC) curve in two ways. First, we analyzed CT IHC index data from all PC vs. all benign samples. The curve yielded an area under the curve (AUC) of $0.9936(\mathrm{P}<0.0001)$ (Fig. 7A). Choosing a cut-off of IHC index of 2.15, a specificity
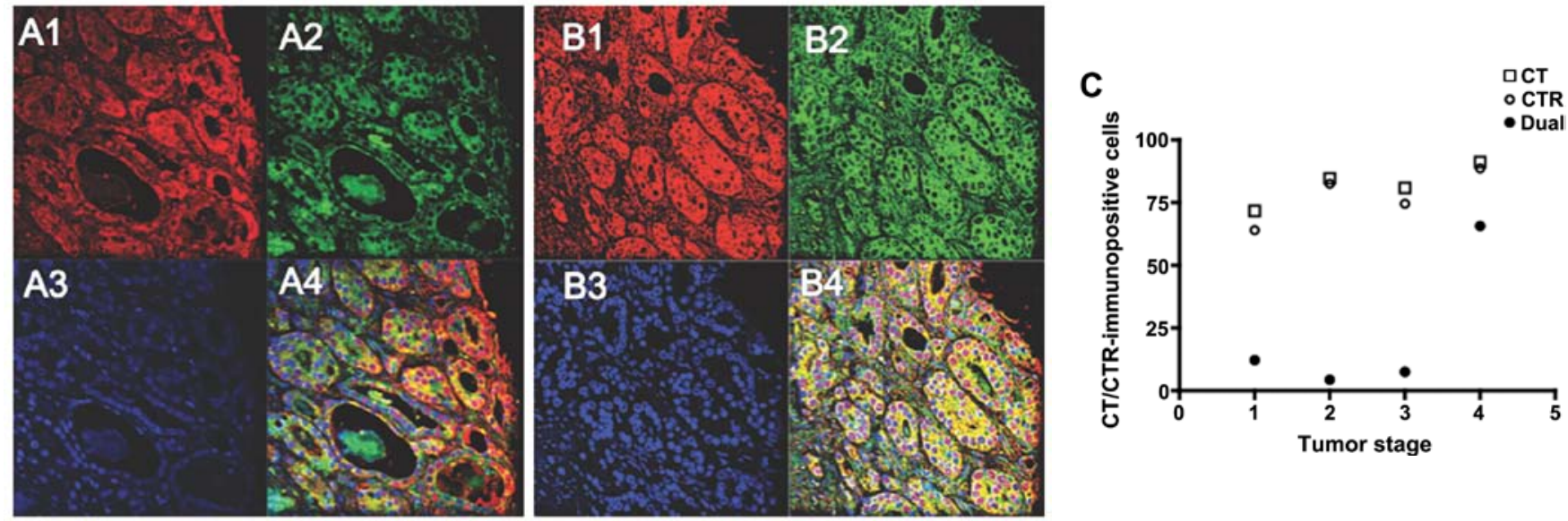

Figure 4. CT-CTR double immunofluorescence. (A and B) PC sections were processed as described in Fig. 1, but after incubation with CT (rabbit) or CTR (mouse) primary antibodies, they were probed with TRITC-conjugated anti-rabbit (red for CT) or FITC-conjugated anti-mouse (green for CTR) secondary antisera. The slides were then counterstained with DAPI (blue for nuclei). Images of every specimen were captured individually with a confocal microscope at $\mathrm{x} 400$. The percentage of positive cells was calculated by dividing the number of positively-stained cells (red or green) with total number of cells (DAPI-stained). 1, CT; 2, CTR; 3, DNA; and 4, merged image. (C) Number of cells expressing CT, CTR and dual (CT+CTR) staining were counted and plotted against $\mathrm{T}$ stage. 

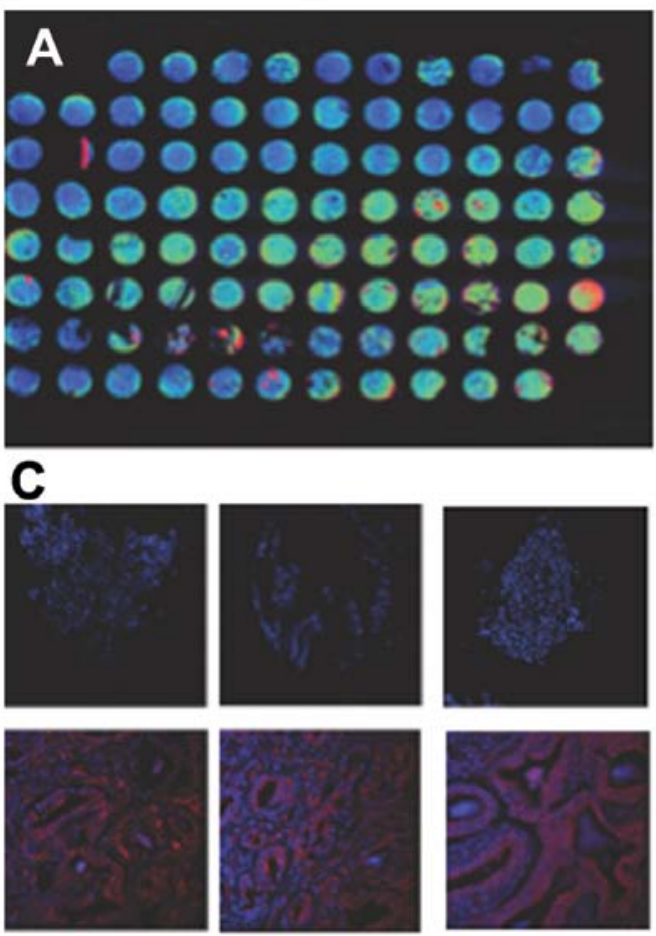
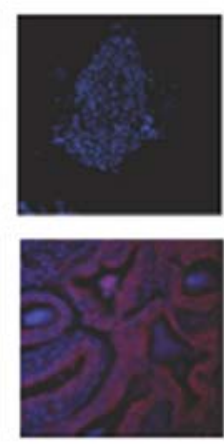

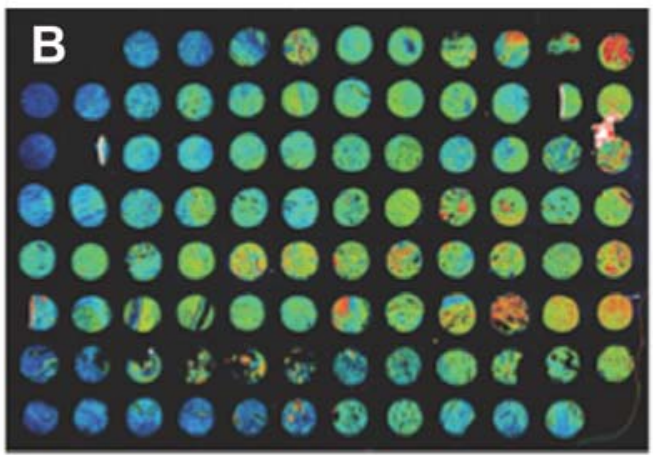

D
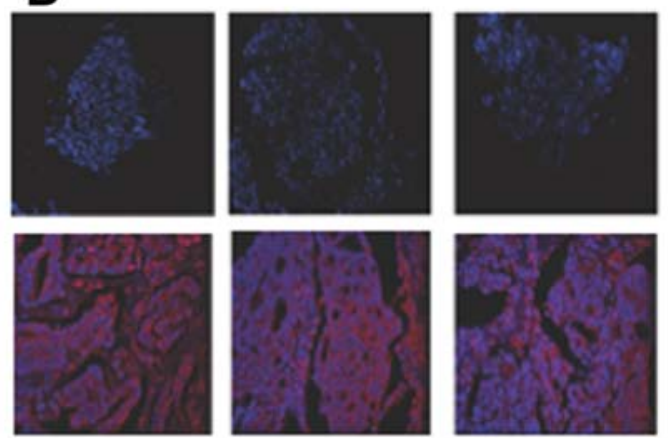

Figure 5. TMA scans and prostate micrographs. TMAs (TMA PR955; US Biomax) were processed as described above for CT or CTR immunofluorescence, and were scanned on a microarray scanner (Perkin-Elmer, Waltham, MA, USA). (A and B) Scans of TMA for CT and CTR respectively. (C and D) Representative micrographs (x400) showing CT/CTR immunostaining (red) and DAPI (blue) in BPH (upper row) and PC specimens (lower row).

Table I. Correlation analysis between CT or CTR expression and clinicopathological parameters.

A, CT expression and clinicopathological parameters

\begin{tabular}{lclc}
\hline Parameter & Pearson's co-efficient & P-value & R-squared \\
\hline Tumor stage & 0.9182 & 0.0049 & 0.8431 \\
Gleason score & 0.9245 & 0.013 & 0.8548 \\
Serum PSA & 1 & 0.1967 & \\
\hline
\end{tabular}

B, CTR expression and clinicopathological parameters

\begin{tabular}{lclc}
\hline Parameter & Pearson's co-efficient & P-value & R-squared \\
\hline Tumor stage & 0.7712 & 0.0383 & 0.5948 \\
Gleason score & -0.8434 & 0.0363 & 0.7114 \\
Serum PSA & 0.5 & 0.5 & \\
\hline
\end{tabular}

of $100 \%$ yielded a sensitivity of $84 \%$, suggesting that CT/CTR IHC index $>2.15$ can reliably discriminate cancer patients from non-cancer patients. Next, we compared the data from localized cancer with that from metastatic cancer. The ROC curve yielded the AUC of $0.6704(\mathrm{P}=0.0264)$ (Fig. 7B). With the cut-off of 9.5 , a specificity of $75 \%$ yielded the sensitivity of 53\% for metastatic disease indicating that the test can be reliably used to predict clinical course of the disease. The corresponding analysis of CTR expression data also showed similar predictability (Fig. 7C and D). Both, CT and CTR IHC indices were better predictors for the cancer when compared with preoperative serum PSA levels; and for metastatic disease when compared with the Gleason score (data not shown).

CT/CTR expression and clinicopathological parameters. We then compared CT IHC indices of benign and PC specimens with preoperative serum PSA levels of the subjects. This is because serum PSA is currently used as a marker for PC. As shown in Fig. 8A and B, CT IHC index as well as preoperative serum PSA levels of this cohort displayed significant discrimination between benign and PC patients. However, the significance of CTR IHC index between these two groups (benign vs. cancer) was higher than that of preoperative serum PSA levels. Next, we compared the ability of CT IHC index and Gleason score to discriminate local vs. metastatic disease (Fig. 8C and D). The analysis shows that CT IHC index significantly discriminated metastatic disease from localized disease, whereas the Gleason score could not. Likewise, CTR IHC index data also demonstrated similar ability (Fig. 8E-H).

Next, contingency analysis of crosstables also identified a significant association between high CT/CTR IHC indices and metastatic cancers (Table IIA and B). Thus, multiple comparative analyses of CT/CTR expression with the established clinicopathological markers/clinical outcome data demonstrate that $\mathrm{CT} / \mathrm{CTR}$ expression could potentially serve as a progonostic marker of metastatic PC.

CT/CTR IHC indices and clinical outcome of prostate cancer patients. Next, we examined the prognostic ability of CT/CTR expression as well as other clinicopathological markers on metastasis-free survival of prostate cancer patients by Kaplan-Meier analysis. Data of patients were divided into 

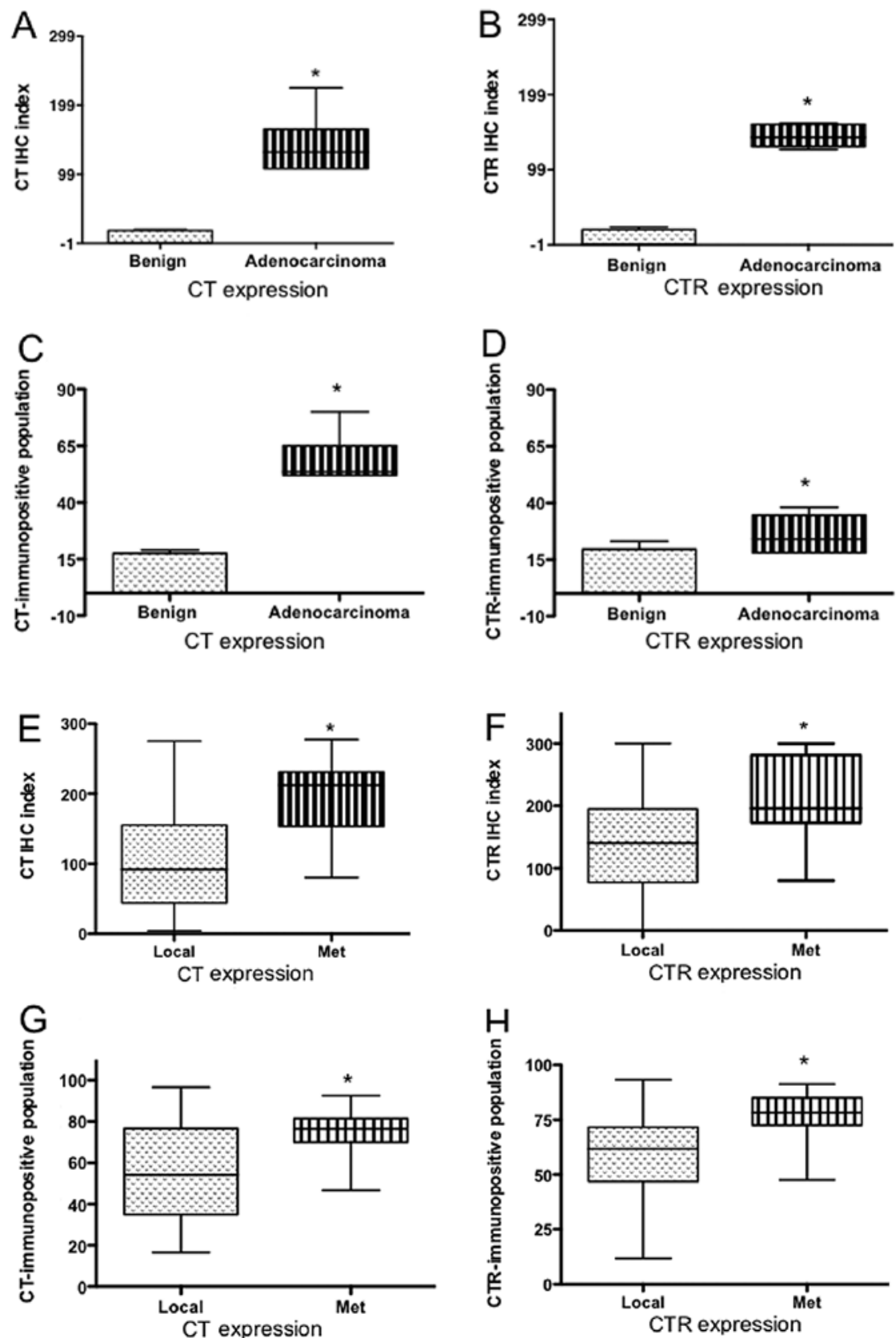

CTR expression
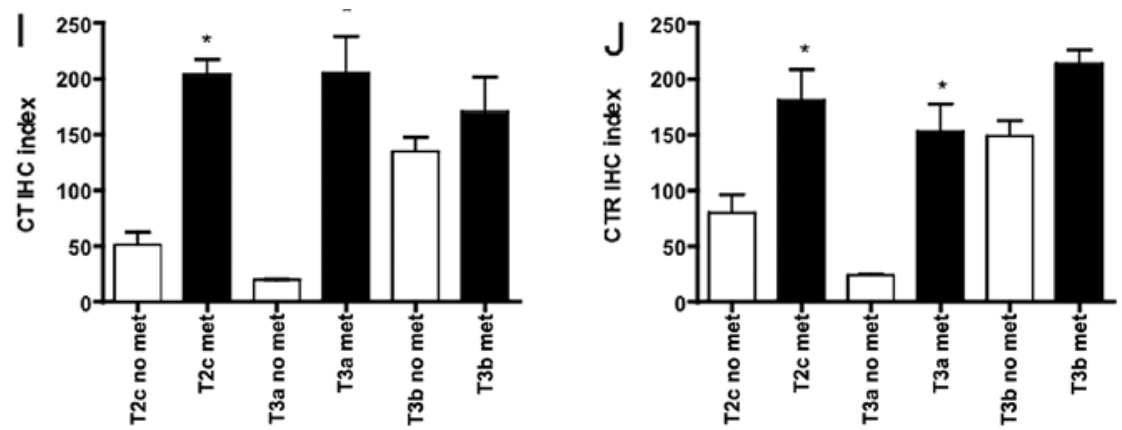

Figure 6. CT and CTR expression in benign vs. adenocarcinoma specimens. (A and B) Box and Whisker plots of CT and CTR IHC indices in benign and malignant prostates. Chi-squared $\mathrm{P}<0.0001$ [ $\mathrm{n}=141$ for $\mathrm{CT}(40,101)$; $\mathrm{n}=141$ for CTR $(40,101)]$. (C and D) Same data are expressed as percent immunopositive (CT or CTR) cells. Chi-squared for CT and CTR: P<0.0001. (E and F) Box and Whisker plots of CT and CTR IHC indices in localized and metastatic PC. The results were analyzed unpaired t-test. $\mathrm{P}<0.0001(\mathrm{n}=101)$ for $\mathrm{CT}$ and $\mathrm{P}<0.0001(\mathrm{n}=101)$ for $\mathrm{CTR}$. (G and H) Same data (as in E-F) are expressed as percent immunopositive (CT or CTR) cells. The results were analyzed unpaired t-test. P<0.0001 ( $\mathrm{n}=101)$ for CT as well as CTR. (I) CT expression was stratified in tumor (T) stages T2c, T3a and T3b (non-met vs. met). The data were analyzed by unpaired t-test. T2c (non-met) vs. T2c (met): P=0.0007 (n=21). T3a (non-met) vs. T3a (met): $\mathrm{P}=0.0286(\mathrm{n}=19)$. T3b (non-met) vs. T3b (met): $\mathrm{P}=0626(\mathrm{n}=48)$, not significant. $(\mathrm{J})$ CTR expression data were further stratified in tumor stages T2c, T3a and T3b (non-met vs. met). The data were analyzed unpaired t-test. T2c (non-met) vs. T2c (met): P=0.0079 (n=21). T3a (non-met) vs. T3a (met): $\mathrm{P}=0.0264$ ( $\mathrm{n}=19)$. T3b (non-met) vs. T3n (met): $\mathrm{P}=0.0259(\mathrm{n}=48)$. 

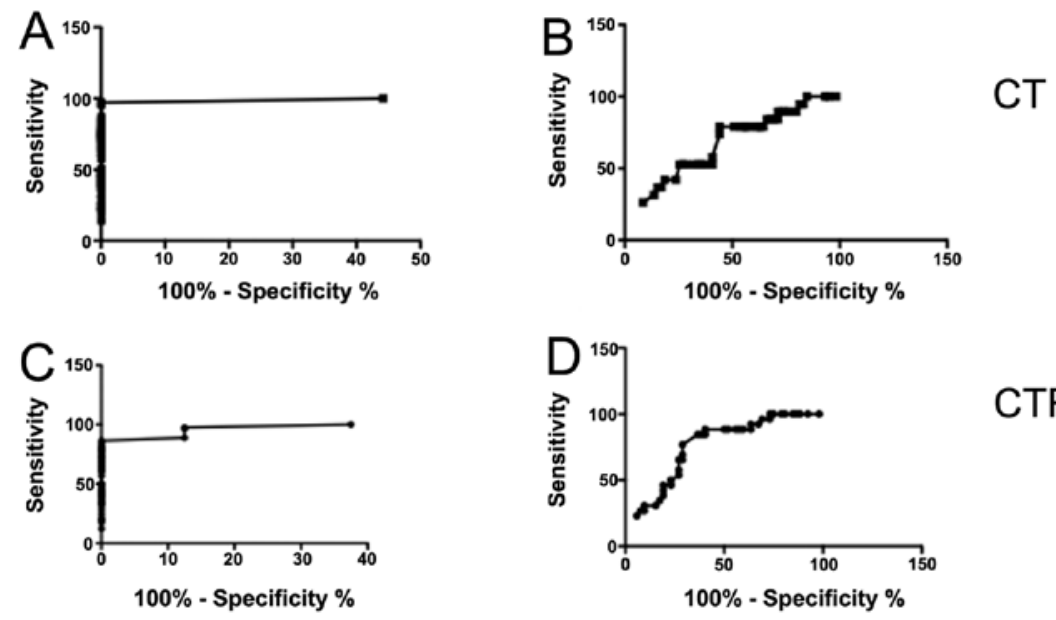

CTR

Figure 7. ROC Curves for CT and CTR expression (IHC index): ROC curves for CT expression were drawn from the data of all prostate specimens studied (benign vs. PC; $\mathrm{P}<0.0001$, curve A); and for CT expression in PC specimens (localized vs. metastatic PC; $\mathrm{P}<0.02624$, curve B). The respective ROC curves for CTR expression are presented in curves $\mathrm{C}(\mathrm{P}<0.0001)$ and $\mathrm{D}(\mathrm{P}<0.0002)$.
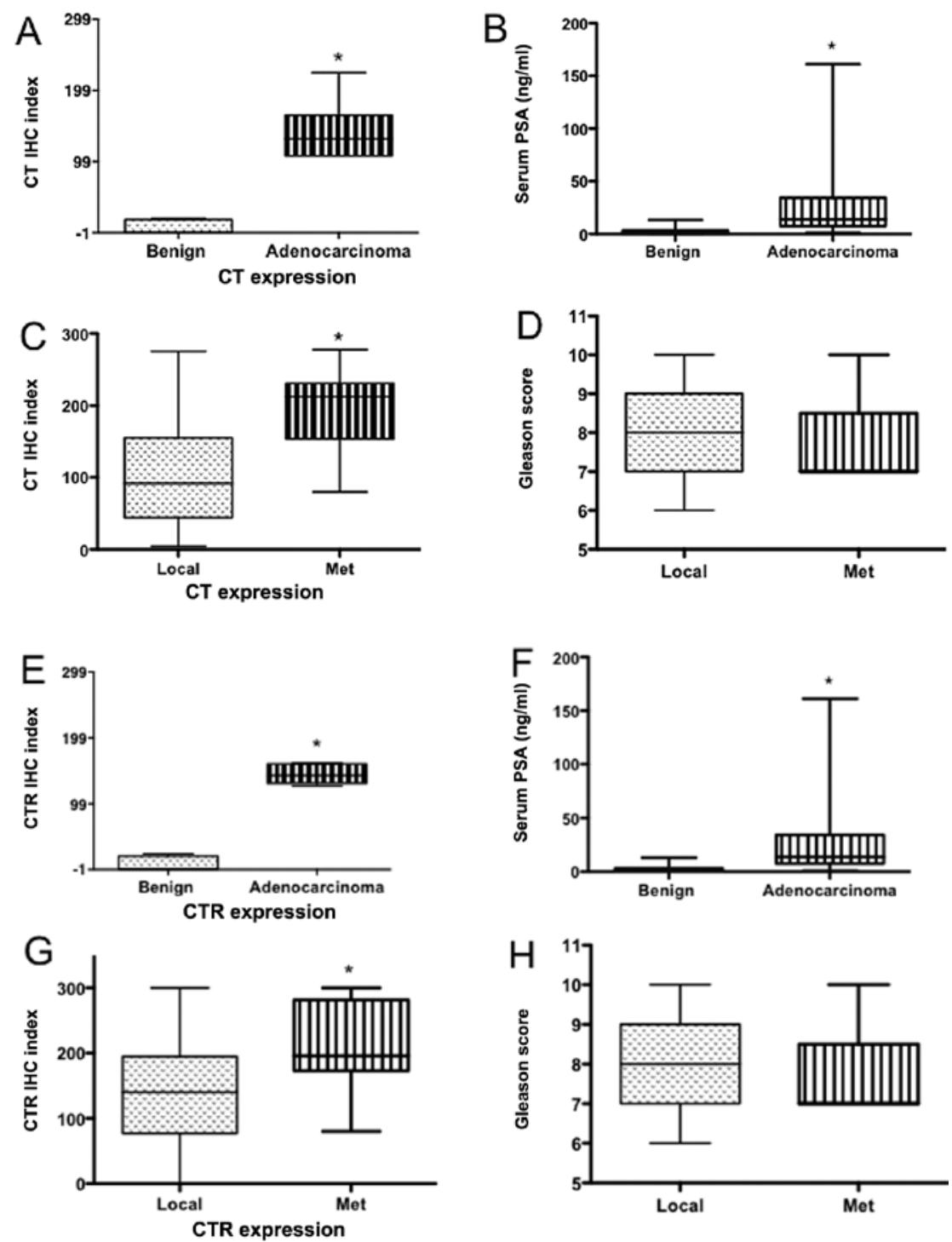

Figure 8. CT/CTR expression and clinicopathological markers for PC and metastatic disease. (A and B) Box and Whisker plots of CT IHC index and preoperative serum PSA levels of the cohort. CT-P $<0.0001$ ( $n=141$, two-tailed t-test); PSA-P=0.0006 ( $\mathrm{n}=137$, two tailed $\mathrm{t}$-test). (C and D) Box and Whisker plots of CT IHC index and Gleason scores of localized and malignant prostate cancer. CT-P=0.0001 ( $n=101$, two tailed t-test); Gleason score: $P=0.0725$ ( $n=101$, two tailed t-test). ( $\mathrm{E}$ and F) Box and Whisker plots of CTR IHC index in benign and preoperative serum PSA levels in patients with benign and malignant prostates. CTR-P $<0.0001$ ( $n=141$, two-tailed t-test); PSA-P=0.0006 ( $n=137$, two tailed t-test). ( $\mathrm{G}$ and H) Box and Whisker plots of CTR IHC index and Gleason scores of localized and malignant prostate cancer. CTR-P=0.0003 ( $\mathrm{n}=101$, two tailed $\mathrm{t}$-test); Gleason score: $\mathrm{P}=0.0725$ ( $\mathrm{n}=101$, two tailed $\mathrm{t}$-test). 
Table II. Crosstables showing CT or CTR expression (cases and percentage of cases in each category) in relation with clinicopathological parameters.

A, CT expression

\begin{tabular}{|c|c|c|c|c|}
\hline Variables & Total patients $(\%)$ & $\begin{array}{c}\text { Low CT IHC } \\
\text { index }(\leq 150)(\%)\end{array}$ & $\begin{array}{c}\text { High CT IHC } \\
\text { index }(>150)(\%)\end{array}$ & P-value \\
\hline \multicolumn{5}{|l|}{ Age (years) } \\
\hline$>66$ & $43(100)$ & $20(47)$ & $23(53)$ & \\
\hline$\leq 66$ & $43(100)$ & $18(42)$ & $25(58)$ & 0.8256 \\
\hline \multicolumn{5}{|c|}{ Preoperative serum PSA (ng/ml) } \\
\hline$\leq 10$ & $22(100)$ & $15(68)$ & $7(32)$ & \\
\hline$>10$ & $61(100)$ & $29(48)$ & $32(52)$ & 0.4626 \\
\hline \multicolumn{5}{|l|}{ pT status } \\
\hline pT2 & $32(100)$ & $17(53)$ & $15(47)$ & \\
\hline pT3 & $49(100)$ & $26(53)$ & $23(47)$ & \\
\hline pT4 & $4(100)$ & $0(0)$ & $4(100)$ & 0.1238 \\
\hline \multicolumn{5}{|l|}{ Gleason score } \\
\hline $5-6$ & $9(100)$ & $6(66)$ & $3(34)$ & \\
\hline 7 & $32(100)$ & $14(44)$ & $18(56)$ & \\
\hline $8-10$ & $44(100)$ & $25(57)$ & $19(43)$ & 0.3630 \\
\hline \multicolumn{5}{|c|}{ Metastatic events } \\
\hline No metastasis & $64(100)$ & $38(59)$ & $26(41)$ & \\
\hline Metastasis & $21(100)$ & $6(29)$ & $15(71)$ & 0.0225 \\
\hline
\end{tabular}

B, CTR expression

\begin{tabular}{|c|c|c|c|c|}
\hline Variables & Total patients $(\%)$ & $\begin{array}{c}\text { Low CT IHC } \\
\text { index }(\leq 150)(\%)\end{array}$ & $\begin{array}{c}\text { High CT IHC } \\
\text { index }(>150)(\%)\end{array}$ & P-value \\
\hline \multicolumn{5}{|l|}{ Age (years) } \\
\hline$>66$ & $37(100)$ & $15(41)$ & $22(59)$ & \\
\hline$\leq 66$ & $42(100)$ & $17(40)$ & $25(60)$ & 1.000 \\
\hline \multicolumn{5}{|c|}{ Preoperative serum PSA (ng/ml) } \\
\hline$\leq 10$ & $21(100)$ & $8(38)$ & $13(62)$ & \\
\hline$>10$ & $58(100)$ & $24(41)$ & $34(59)$ & 1.000 \\
\hline \multicolumn{5}{|l|}{ pT status } \\
\hline $\mathrm{pT} 2$ & $26(100)$ & $15(58)$ & $11(42)$ & \\
\hline pT3 & $49(100)$ & $17(35)$ & $32(65)$ & \\
\hline pT4 & $4(100)$ & $1(25)$ & $3(75)$ & 0.1236 \\
\hline \multicolumn{5}{|l|}{ Gleason score } \\
\hline $5-6$ & $7(100)$ & $4(57)$ & $3(43)$ & \\
\hline 7 & $30(100)$ & $15(50)$ & $15(50)$ & \\
\hline $8-10$ & $41(100)$ & $15(37)$ & $26(63)$ & 0.3324 \\
\hline \multicolumn{5}{|c|}{ Metastatic events } \\
\hline No metastasis & $52(100)$ & $31(60)$ & $21(40)$ & \\
\hline Metastasis & $26(100)$ & $6(23)$ & $20(77)$ & 0.0321 \\
\hline
\end{tabular}

two, high $(>150)$ and low $(\leq 150)$ CT/CTR IHC index groups; and survival period of the patients was considered as a clinical outcome. Likewise, the patients were also analyzed for other clinicopathological parameters as described in Fig. 9. Patients with high CT/CTR expression demonstrated significantly greater unfavorable disease course than those with low CT/CTR expression ( $\mathrm{P}<0.0005$; Fig. 9A and B). Among other clinicopathological parameters, only Gleason score showed 

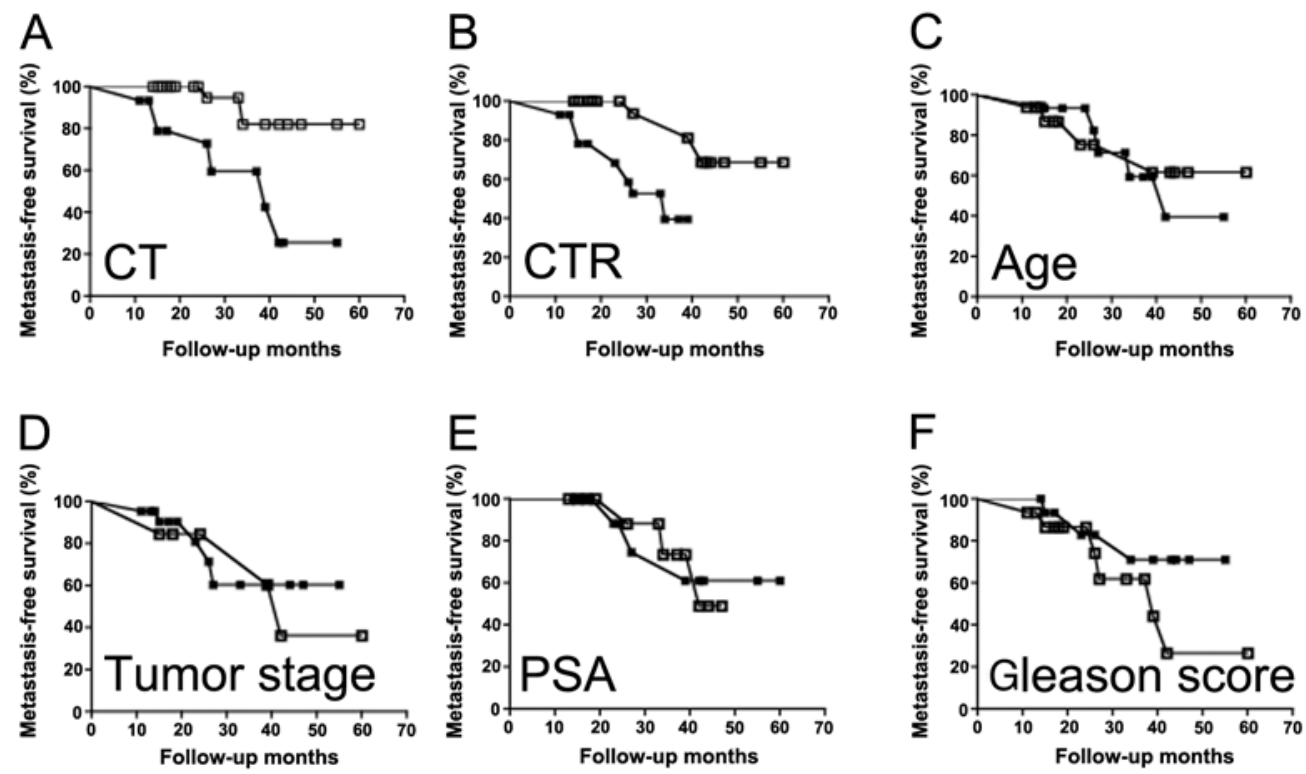

Figure 9. Kaplan-Meier survival curves. (A) Survival curve in patients with PC based on the mean values of CT IHC index in tumor tissues, the cases were divided into two groups: high $(>150)$ and low $(\leq 150)$ expression. Overall and metastasis-free survival curve in total 101 tumors. P-value was analyzed by log-rank test. Additional data analysis is presented in Tables III and IV. (B) Survival curve of patients with PC based on the mean values of CTR IHC index in tumor tissues, the cases were divided into two groups at this level: high and low expression. Overall and metastasis-free survival curve in a total of 101 tumors. P-value was analyzed by log-rank test. Additional data analysis is presented in Tables III and IV. (C) Survival curve of patients with PC based on the age of the patients, the cases were divided into two groups at this level: < or $>66$ years. Overall and metastasis-free survival curve in a total of 101 tumors. P-value was analyzed by log-rank test. Additional data analysis is presented in Tables III and IV. (D) Survival curve of patients with PC based on the tumor stage of the patients, the cases were divided into two groups at this level: T stage II or T stage III. Overall and metastasis-free survival curve in a total of 101 tumors. P-value was analyzed by log-rank test. Additional data analysis is presented in Tables III and IV. (E) Survival curve of patients with PC based on preoperative serum PSA levels, the cases were divided into two groups at this level: high and low levels. Overall and metastasis-free survival curve in a total of 101 tumors. P-value was analyzed by log-rank test. Additional data analysis is presented in Tables III and IV. (F) Survival curve of patients with PC based on the Gleason score of the tumor tissues, the cases were divided into two groups at this level: $<$ or $>8$. Overall and metastasis-free survival curve in a total of 101 tumors. P-value was analyzed by log-rank test. Additional data analysis is presented in Tables III and IV.

Table III. Metastasis-free Kaplan-Meir survival curve log-rank test for CT and CTR expression.

\section{A, CT expression}

\begin{tabular}{lccccc}
\hline Parameter & Chi-square & P-value & Median survival-high & Median survival-low & Hazard ratio \\
\hline CT & 12.41 & 0.0004 & 39 & Undefined & 6.72 \\
PSA & 0.6330 & 0.4262 & 42 & Undefined & 1.637 \\
Age & 1.186 & 0.2761 & 39 & Undefined & 1.89 \\
Tumor stage & 0.1291 & 0.7194 & 42 & Undefined & 1.193 \\
Gleason score & 5.646 & 0.0176 & Undefined & 38 & 0.2854 \\
\hline
\end{tabular}

B, CTR expression

\begin{tabular}{llcccc}
\hline Parameter & Chi-square & P-value & Median survival-high & Median survival-low & Hazard ratio \\
\hline CTR & 12.02 & 0.0005 & 34 & Undefined & 4.672 \\
PSA & 0.001188 & 0.3724 & Undefined & 42 & 0.3808 \\
Age & 0.1261 & 0.7236 & 42 & Undefined & 1.177 \\
Tumor stage & 0.2326 & 0.6289 & 42 & Undefined & 1.27 \\
Gleason score & 3.672 & 0.0491 & 33 & Undefined & 2.52 \\
\hline
\end{tabular}

prognostic significance. However, the level of significance was remarkably lower than that of $\mathrm{CT} / \mathrm{CTR}$ expression $[\mathrm{P}=0.0491$ vs. $\mathrm{P}=0.0005$ (for $\mathrm{CT} / \mathrm{CTR}$ ), Fig. 9F]. All other parameters failed the test (Fig. 9C-E; Table IIIA and B). We further tested the data with Cox regression analysis. In a stratified univariate analysis, a highly significant prognostic value for high CT/CTR 
Table IV. Metastasis-free survival and clinicopathological paradigms.

\begin{tabular}{lccccccr}
\hline & \multicolumn{3}{c}{ Univariate } & & \multicolumn{3}{c}{ Multivariate } \\
\cline { 2 - 3 } Paradigm & Hazard ratio & $95 \%$ CI & P-value & & Hazard ratio & $95 \%$ CI & P-value \\
\hline CT & 6.342 & $2.269-17.73$ & 0.0004 & & 6.284 & $1.402-28.167$ & 0.016 \\
CTR & 6.237 & $2.216-17.56$ & 0.0005 & & 9.813 & $2.201-43.742$ & 0.003 \\
PSA & 1.682 & $0.4673-5.034$ & 0.4262 & & & \\
Age & 1.781 & $0.6303-5.034$ & 0.2761 & & & 0.410 \\
Tumor stage & 1.221 & $0.4106-3.632$ & 0.7194 & & & & \\
Gleason score & 0.2946 & $0.1075-0.8073$ & 0.0175 & & 0.571 & $0.151-2.165$ & \\
\hline
\end{tabular}

expression was found with lower survival period; whereas in cases with low CT/CTR levels, the survival period was longer (Table IV). Among other parameters, only Gleason score displayed similar prognostic prediction with a lower significance. In multivariate Cox analyses, CT/CTR expression and Gleason scores showed significant prognostic values. Based on this analysis, CT/CTR IHC index is a significantly better prognostic indicator than Gleason score, preoperative serum PSA, age, or tumor stage (Table IV).

\section{Discussion}

Present results demonstrate that both, CT and CTR, are selectively localized in basal epithelium of benign prostate acinii. However, this spatial specificity seems lost in PC, as indicated by staining of CT and CTR staining in whole malignant pseudoacinii, which lack basal cells. These results are consistent with our earlier study on localization of CT/CTR mRNA expression in the prostate, and validate the reagents and procedures used in the present study (9). We also observed that CT/CTR expression was elevated in HGPIN sections as well as malignanant epithelia. Considering that HGPINs are shown to represent premalignant lesions that may later develop into neoplasms, the results raise a possibility that CT-CTR axis may be among early genes activated during neoplastic transformation of the prostate (18).

Present results have also identified several new aspects of CT/CTR expression. First, CT/CTR-immunopositive expression in metastatic PCs was remarkably higher even during earlier tumor stages as compared to that in non-metastatic ones. Second, much larger cell populations of metastatic PCs co-expressed CT and CTR than non-metastatic ones. Third, CT was abundantly stained in bone metastasis of PC. When combined with our earlier results that the activation of CT-CTR autocrine axis significantly increases tumorigenic capacity and metastasizing ability of multiple PC cell lines $(8,14)$, present results lend pathological significance to our molecular studies on metastasis of prostate cancer. Autocrine CT-CTR axis may promote tumor metastasis through multiple actions including potent chemotactic actions, destabilization of cell-cell junctions, loss of cell-cell adhesion and activation of PI3K-Akt-survivin, cyclic AMP and Wnt- $\beta$-catenin signaling pathways $(17,19-21)$. The patterns of CT/CTR expression observed in clinical samples such as high CT/CTR expression in earlier $\mathrm{T}$ stages and the switching of CT-CTR expression from paracrine to autocrine may prove useful in differentiating metastatic tumors from non-metastatic ones. It is important to note that numerous studies have reported genomic alterations as well as elevated expression of CT/CTR genes in several human malignancies $(3-5,9,14,20,22-26)$. This evidence further reinforces the potential role for CT-CTR axis in pathology of prostate cancer progression.

There is an ongoing search for prognostic biomarkers in $\mathrm{PC}$ as currently used markers are limited in predicting disease outcome (27-29). Our results suggest that PCs with high CT/ CTR expression showed a tendency towards a worse course (lower incidence of metastasis-free survival) than the patients with low CT/CTR expression. Gleason score also provided similar predictive value, but was less reliable than CT/CTR in these cohorts. This led us to hypothesize the following interdependencies. First, transformation of benign prostate to a malignancy is associated with the loss of spatial specificity and upregulation of CT/CTR expression. Second, the specimens from metastatic PC displayed a switch of CT-CTR axis from a paracrine to an autocrine one. Similar phenomenon has been shown to occur in case of androgens as well (30). Third, high level of CT/CTR expression is correlated with poor prognosis and a higher probability of metastatic disease. Among various clinicopathological factors studied, CT/CTR expression provided best predictability of the clinical course. In conclusion, the present data support the use of CT/CTR as therapeutic targets for aggressive $\mathrm{PC}$ and provide a strong rationale for a larger study to evaluate $\mathrm{CT} / \mathrm{CTR}$ as prognostic markers for metastatic PC.

\section{References}

1. Horwich A, Parker C and Kataja V: Prostate cancer: ESMO clinical recommendations for diagnosis, treatment and follow-up. Ann Oncol 20 (Suppl 4): S76-S78, 2009.

2. Armstrong AJ and Febbo PG: Using surrogate biomarkers to predict clinical benefit in men with castration-resistant prostate cancer: an update and review of the literature. Oncologist 14: 816-827, 2009.

3. Deng B, Zhang S, Miao Y, et al: Adrenomedullin expression in epithelial ovarian cancers and promotes HO8910 cell migration associated with upregulating integrin alpha5beta1 and phosphorylating FAK and paxillin. J Exp Clin Cancer Res 31: 19, 2012.

4. Wang X, Nakamura M, Mori I, et al: Calcitonin receptor gene and breast cancer: quantitative analysis with laser capture microdissection. Breast Cancer Res Treat 83: 109-117, 2004.

5. Delis S, Bakoyiannis A, Giannakou N, Tsigka A, Avgerinos C and Dervenis C: Asymptomatic calcitonin-secreting tumor of the pancreas. A case report. JOP 7: 70-73, 2006. 
6. Ferrigno D, Buccheri G and Biggi A: Serum tumour markers in lung cancer: history, biology and clinical applications. Eur Respir J 7: 186-197, 1994

7. Shah GV, Noble MJ, Austenfeld M, Weigel J, Deftos LJ and Mebust WK: Presence of calcitonin-like immunoreactivity (iCT) in human prostate gland: evidence for iCT secretion by cultured prostate cells. Prostate 21: 87-97, 1992.

8. Thomas S, Chigurupati S, Anbalagan M and Shah G: Calcitonin increases tumorigenicity of prostate cancer cells: evidence for the role of protein kinase A and urokinase-type plasminogen receptor. Mol Endocrinol 20: 1894-1911, 2006.

9. Chien J, Ren Y, Qing Wang Y, et al: Calcitonin is a prostate epithelium-derived growth stimulatory peptide. Mol Cell Endocrinol 181: 69-79, 2001

10. Conner AC, Simms J, Hay DL, et al: Heterodimers and family-B GPCRs: RAMPs, CGRP and adrenomedullin. Biochem Soc Trans 32: 843-846, 2004.

11. Purdue BW, Tilakaratne N and Sexton PM: Molecular pharmacology of the calcitonin receptor. Receptors Channels 8: 243-255, 2002 .

12. Tolcos M, Tikellis C, Rees S, Cooper M and Wookey P: Ontogeny of calcitonin receptor mRNA and protein in the developing central nervous system of the rat. J Comp Neurol 456: 29-38, 2003

13. Wookey PJ, McLean CA, Hwang P, et al: The expression of calcitonin receptor detected in malignant cells of the brain tumour glioblastoma multiforme and functional properties in the cell line A172. Histopathology 60: 895-910, 2012.

14. Shah GV, Thomas S, Muralidharan A, et al: Calcitonin promotes in vivo metastasis of prostate cancer cells by altering cel signaling, adhesion, and inflammatory pathways. Endocr Relat Cancer 15: 953-964, 2008.

15. Chigurupati S, Kulkarni T, Thomas S and Shah G: Calcitonin stimulates multiple stages of angiogenesis by directly acting on endothelial cells. Cancer Res 65: 8519-8529, 2005.

16. Jackel MC, Mitteldorf C, Schweyer S and Fuzesi L: Clinical relevance of Fas (APO-1/CD95) expression in laryngeal squamous cell carcinoma. Head Neck 23: 646-652, 2001.

17. Shah GV, Muralidharan A, Gokulgandhi M, Soan K and Thomas S: Cadherin switching and activation of $\beta$-catenin signaling underlie proinvasive actions of calcitonin-calcitonin receptor axis in prostate cancer. J Biol Chem 284: 1018-1030, 2009 .
18. Brawer MK: Prostatic intraepithelial neoplasia: an overview. Rev Urol 7 (Suppl 3): S11-S18, 2005.

19. Thomas $\mathrm{S}$ and Shah G: Calcitonin induces apoptosis resistance in prostate cancer cell lines against cytotoxic drugs via the Akt/survivin pathway. Cancer Biol Ther 4: 1226-1233, 2005.

20. Ritchie CK, Thomas KG, Andrews LR, Tindall DJ and Fitzpatrick LA: Effects of the calciotrophic peptides calcitonin and parathyroid hormone on prostate cancer growth and chemotaxis. Prostate 30: 183-187, 1997.

21. Shah GV, Rayford W, Noble MJ, et al: Calcitonin stimulates growth of human prostate cancer cells through receptormediated increase in cyclic adenosine 3',5'-monophosphates and cytoplasmic $\mathrm{Ca}^{2+}$ transients. Endocrinology 134: 596-602, 1994.

22. Goodman MT, Ferrell R, McDuffie K, et al: Calcitonin gene polymorphism CALCA-624 (T/C) and ovarian cancer. Environ Mol Mutagen 46: 53-58, 2005.

23. Trimboli P, Rossi F, Baldelli R, et al: Measuring calcitonin in washout of the needle in patients undergoing fine needle aspiration with suspicious medullary thyroid cancer. Diagn Cytopathol 40: 394-398, 2012

24. Hebden C, Smalt R, Chambers T and Pondel MD: Multiple promoters regulate human calcitonin receptor gene expression. Biochem Biophys Res Commun 272: 738-743, 2000.

25. Silverman AL, Park JG, Hamilton SR, Gazdar AF, Luk GD and Baylin SB: Abnormal methylation of the calcitonin gene in human colonic neoplasms. Cancer Res 49: 3468-3473, 1989.

26. Shah GV: Calcitonin. Encyclopedia of Cancer 2: 16-20, 2009.

27. Yigitbasi O, Ozturk U, Goktug HN, Gucuk A and Bakirtas H: Prognostic factors in metastatic prostate cancer. Urol Oncol 29: 162-165, 2011.

28. Andren O, Fall K, Franzen L, Andersson SO, Johansson JE and Rubin MA: How well does the Gleason score predict prostate cancer death? A 20-year followup of a population based cohort in Sweden. J Urol 175: 1337-1340, 2006.

29. Masieri L, Lanciotti M, Gontero P, et al: The prognostic role of preoperative chromogranin A expression in prostate cancer after radical prostatectomy. Arch Ital Urol Androl 84: 17-21, 2012.

30. Gao J, Arnold JT and Isaacs JT: Conversion from a paracrine to an autocrine mechanism of androgen-stimulated growth during malignant transformation of prostatic epithelial cells. Cancer Res 61: 5038-5044, 2001 